Bot. GAZ. 142(3):394-401. 1981.

(c) 1981 by The University of Chicago. 0006-8071/81/4203-0015 $\$ 02.00$

\title{
BIFURCATION RATIOS AND THE ADAPTIVE GEOMETRY OF TREES
}

\author{
ROLF BORCHERT AND NORMAN A. SLADE \\ Division of Biological Sciences, University of Kansas, Lawrence, Kansas 66045
}

\begin{abstract}
In several recent studies the branching system of trees was assumed to constitute a geometric series with constant branching ratio indicative of the adaptive geometry of a species. How ever, in a population of young cottonwood trees (Populus deltoides), branching ratios varied significantly within the crown of individual trees and among experimental groups of different growth vigor. With a simple mathematical model, based on the symmetric branch system of the tropical tree Tabebuia rosea, we showed that, with increasing size of a tree, the geometric (exponential) increase in branch number per order must be reduced, if a physiologically optimum leaf area per terminal branch is to be maintained. Also, branching ratios based on centripetal branch ordering are not predictive of tree development and ignore information essential to characterize the architectural model of a tree. Bifurcation ratios thus appear to be unsuited as indicators of the adaptive geometry of trees.
\end{abstract}

\section{Introduction}

During the past decade interest in the mathematical analysis of branched biological systems such as botanical trees or bronchial, arterial, and neural dendritic trees (Uyings, SMITh, and Veltman 1975) has risen dramatically. Mathematical analysis of botanical trees should shed light on the following aspects of tree biology:

1. Trees grow by adding relatively few structural elements-stems, buds, leaves, and roots-to the existing organs over prolonged periods. This development is not deterministic but strongly affected by a variety of internal and environmental factors and results in great phenotypic variation among trees of the same species. Nevertheless, an experienced observer will recognize many tree species at first glance because of their specific architecture. The diversity of tree branching probably exceeds that of all other biological branching systems (HALLÉ, OLDEMAN, and TomLinson 1978), but except for HondA's (1971) method of describing tree form in terms of branching angle and branch (segment) length, mathematical methods that adequately characterize tree architecture do not exist.

2. To understand the basic function of botanical trees as devices to capture solar energy, we need to establish correlations between the structure of a branch system and its function to provide mechanical support for leaves and transport channels among the organs of a tree. To this end, quantification of photosynthetic surfaces, of branch dimensions which determine transport resistances and mechanical requirements, branching angles, and other parameters of tree architecture is needed (e.g., McMAHON and KronaUer 1976; ZimmermanN 1978).

3. The mathematical analysis of tree architecture

Address for correspondence and reprints: ROLF BORCHERT, Division of Biological Sciences, University of Kansas, Lawrence, Kansas 66045.

Manuscript received November 1980; revised manuscript received February 1981. should ultimately aid in understanding the optimization of the structure-function relationship for a certain set of environmental conditions, i.e., the "adaptive strategy" of trees. While this analysis has been attempted for the relatively small number of growth patterns observed in temperate trees (HORN 1971), extension of such studies to the great structural variety observed in tropical trees (HALLÉ et al. 1978) will be a formidable task.

In most recent morphometric studies of botanical trees, bifurcation ratios have been used to characterize tree architecture; but a basic study demonstrating that this form of mathematical analysis is biologically appropriate is missing. Based on morphometric data of young, plantation-grown cottonwood (Populus deltoides) and observations of the tropical tree Tabebuia rosea, we show here that, for a number of practical and theoretical reasons, this method is not a satisfactory tool to characterize tree architecture.

\section{Bifurcation ratios and the ordering of branch systems}

There are two principal ways to order segments or branches in a branch system: centrifugal or centripetal ordering (UvLINGS et al. 1975). Botanists have traditionally ordered trees centrifugally by assigning order no. 1 to the main stem and increasing order numbers in consecutive lateral branches (fig. 1) (WIISON 1966). Such centrifugal ordering systems are isomorphic with tree development (see no. 1 in Introduction) and thus meet a basic prerequisite of any mathematical model (MILLER 1978). Increasing order numbers reflect the chronological order of branch formation, and the dynamics of a branching system can be described by adding segments of higher order number after consecutive growth flushes. This ordering system has the disadvantage that branch segments of identical positional and functional value, such as terminal, leaf-bearing segments, may carry widely different order numbers (fig. 1). 
In centripetal ordering systems (STRAHLER 1957; UYLINGs et al. 1975), ordering is initiated at terminal, distal branches, and (in the Strahler system) order number is increased when two branches of equal order meet (fig. 1). The Strahler system was developed for the mathematical description of river systems, which result from connecting a number of peripheral sources. The dynamics of such systems is, thus, opposite to that of tree development. While centripetal ordering systems are isomorphic with rivers, they are not with botanical trees. They group functionally equivalent, terminal tree segments into the same order (fig. 1), but are unsuited to describe the dynamics of tree development because any addition of new terminal branches during tree growth changes order numbers of older segments. Addi-

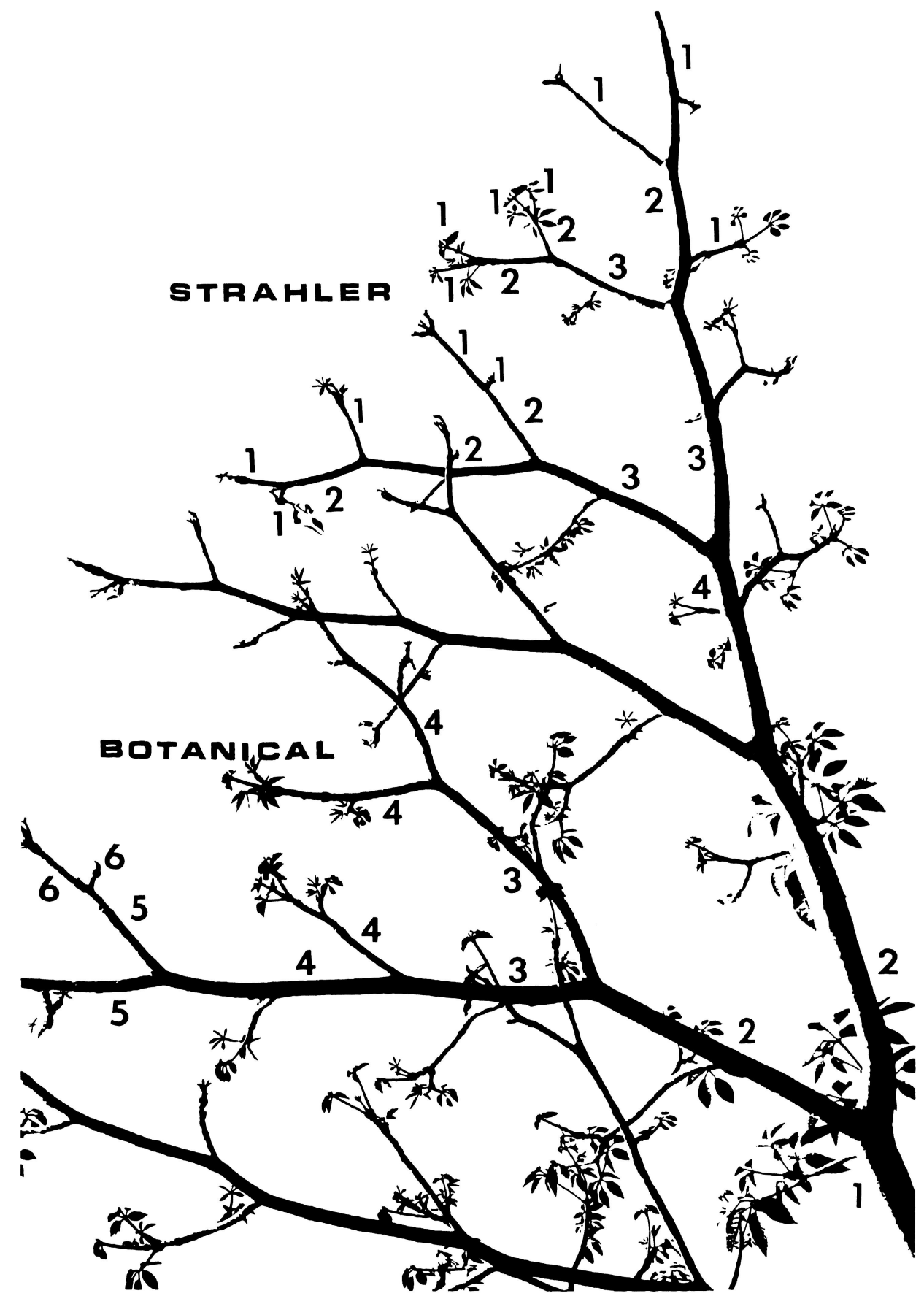

FIG. 1.-Branch system of the tree Tabebuia rosea (Bignoniaceae). Centripetal ordering by the Strahler method is illustrated in the upper half of the figure (numbers on branch segments), and centrifugal ordering by the botanical (natural) method in the lower half. 
tional properties of centrifugal and centripetal ordering systems were discussed by UyLINGs et al. (1975). Throughout this study, Strahler orders will be referred to as $N, \mathrm{I}, \mathrm{II}$, etc.; and botanical order numbers as $n, 1,2$, etc.

The concept of the bifurcation or branching ratio $(R b)$ rests on HoRToN's (1945) suggestion that, in a drainage network, the number of streams (segments) of each order forms an inverse geometric series with order number, which is constant throughout a river system. This concept was applied to botanical trees with the explicit assumption that $R b$ is constant throughout the branching system (Оoнata and Shider 1971; Tominnson 1978). Based on this, so far unproven, assumption, $R b$ can be obtained:

1 . by counting all segments $(S)$ of any two consecutive Strahler orders $(N, N+1)$ and calculating

$$
R b=S_{N} / S_{N+1}
$$

2 . by counting the number of segments in each order of a representative sample of a large tree or an entire small tree and plotting the logarithm of the number of segments in each order $\left(\log S_{N}\right)$ against order $(N)$ (LEOPOLD 1971; BARKER, CUMMING, and HoRSFIELD 1973) (fig. 2). If $R b$ is constant throughout the tree, this plot will yield a straight line, and the regression coefficient of the line of best fit through the values is the negative antilog of $R b$.

When branching occurs by symmetrical dichotomy, $R b=2$. With increasing asymmetry of a Strahlerordered branching system, $R b$ values increase.

\section{Morphometry and bifurcation ratios in cottonwood saplings}

In spring 1976, 1,800 1-yr-old unbranched seedlings of cottonwood (Populus deltoides Batr.), cut to a uniform height of ca. $70 \mathrm{~cm}$, were planted on a 1.6 ha site at the Sunflower Research Area near Lawrence, Kansas. Because of variation in site factors (Borchert, Laushman, and Glass 1981), in fall 1976 saplings in various subsections of the plantation differed significantly in size, and morpho-

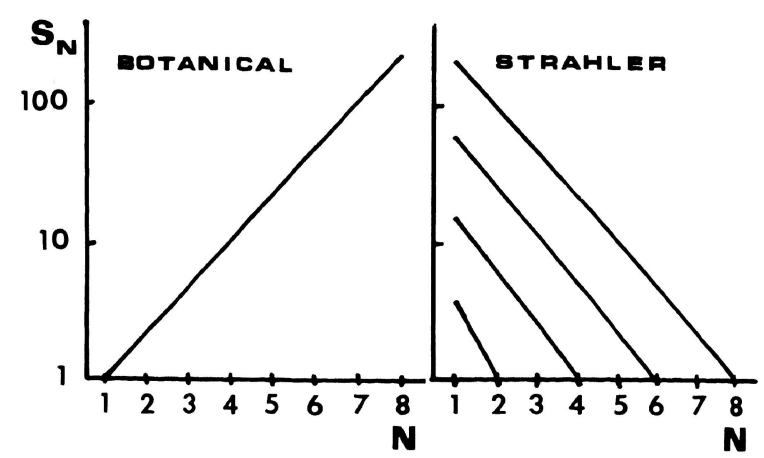

Fig. 2.-Semilogarithmic plot of the theoretical number of branches per order $\left(S_{N}\right)$ vs. order number $(N)$ in the sympodial, symmetrical branch system of Tabebuia rosea; left: botanical; right: Strahler ordering. metric measurements were taken on 10 groups of 10 adjacent trees spaced $1.2 \mathrm{~m}$ apart. Selected morphometric data for six groups of trees (table 1) illustrate the site-dependent variation in morphology and branching ratios within this population of cottonwood saplings.

During the 1976 growing season all saplings formed between one and 12 second-order branches $\left(S_{2}\right.$, table 1$)$, which in turn produced up to 76 thirdorder (proleptic) branches per tree $\left(S_{3}\right.$, table 1$)$ on vigorous saplings. The number and length of all branches were determined, and group means of branch numbers and total length per tree of $S_{2}$ and $S_{3}$ were calculated (table 1). From these data, Strahler orders and bifurcation ratios were obtained as follows:

$$
\begin{array}{ll} 
& S_{\mathrm{I}}=S_{2}+S_{3} ; S_{\mathrm{II}}=S_{2} ; S_{\mathrm{III}}=S_{1}=1 ; \\
\text { and } & R b_{\mathrm{SI}_{\mathrm{I}} \mathrm{SII}}=\left(S_{2}+S_{3}\right) / S_{2} ; R b_{\mathrm{SII} / \mathrm{SII}}=S_{2} .
\end{array}
$$

Statistical analysis showed the following: (a) Based on morphometric data (tree height, stem diameter, crown diameter, and data of table 1), means of all experimental groups were significantly different from each other $(P<.001$; one-way multivariate analysis of variance; HARRIS [1975]). (b) The branching ratios $R b_{S_{I} / S_{\text {II }}}$ and $R b_{S_{\text {II }} / S_{\text {III }}}$ were different from each other when compared for 50 individual trees $(P<.001$; Fisher's sign test; HollaNDER and Wolfe [1973]). (c) In spite of the large withingroup variation (see ranges in table 1 ), there were significant differences for both branching ratios between the groups $(P<.025$; one-way analysis of variance; SoKAL and ROHLF [1969]).

Within the relatively small population of cottonwood saplings, differences in site factors thus caused significant differences in growth vigor. High vigor of trees (groups 1 and 2 in table 1) is indicated by large size (branch length) and complexity (branch number) and by high bifurcation ratios. Conversely, low vigor is indicated by low values of these parameters (group 6, table 1). Bifurcation ratios are thus highly correlated with growth vigor and depend on the environmental conditions affecting tree growth, but vary also within the crown of individual trees. We also observed seasonal changes of $R b_{S_{\mathrm{I}} / \mathrm{SII}_{\mathrm{II}}}$ within tree crowns; up to 22 lateral shoots emerged on vigorous terminal branches of 3-yr-old cottonwood trees (resulting in a $R b_{S_{I} / S_{\text {II }}}$ of 22 on such branches!), but more than half of these laterals had usually died by the end of the growing season ("self-pruning" of trees; Millington and Chaney [1973]).

These observations reinforce the results of other studies: Branching ratios of vigorous, open-grown tree saplings were significantly greater than those of shade-grown saplings (STEINGRAEBER, KASCHT, and Franck 1979; Picketr and KempF 1980), and in oak saplings (Quercus phillaeroides) they varied 
TABLE 1

NUMBER AND LENGTH OF BOTANICAL SECOND- AND THIRD-ORDER BRANCHES OF SIX GROUPS OF 2-YR-OLD COTTONWOOD SAPLINGS (POPULUS DELTOIDES) AND STRAHLER BRANCHING RATIOS CALCULATED FROM THESE DATA ACCORDING TO EQUATIONS (2) AND (3)

\begin{tabular}{|c|c|c|c|c|c|c|}
\hline Group no. & $\begin{array}{c}\text { No. of } \\
S_{2} \text { per tree }\end{array}$ & $\begin{array}{l}\text { Total length of } \\
S_{2} \text { per tree } \\
(\mathrm{cm})\end{array}$ & $\begin{array}{c}\text { No. of } \\
S_{3} \text { per tree }\end{array}$ & $\begin{array}{l}\text { Total length of } \\
S_{3} \text { per tree } \\
(\mathrm{cm})\end{array}$ & $R b_{S \mathbf{S}} / \mathrm{SII}_{\mathrm{I}}$ & $R b_{S \mathrm{SI}} / \mathrm{SIII}$ \\
\hline $\begin{array}{l}1 \ldots \ldots \\
2 \ldots \ldots \\
3 \ldots \ldots \\
4 \ldots \ldots \\
5 \ldots \ldots \\
6 \ldots \ldots\end{array}$ & $\begin{array}{c}7.5 \pm 2.9 \\
(2-12) \\
7.9 \pm 2.5 \\
(5-12) \\
4.9 \pm 2.5 \\
(3-10) \\
5.5 \pm 2.0 \\
(3-10) \\
5.4 \pm 1.4 \\
(3-8) \\
4.5 \pm 1.8 \\
(1-6)\end{array}$ & $\begin{array}{c}864 \pm 355 \\
(274-1407) \\
816 \pm 286 \\
(447-1361) \\
433 \pm 180 \\
(167-683) \\
463 \pm 136 \\
(282-688) \\
416 \pm 108 \\
(261-650) \\
254 \pm 108 \\
(45-406)\end{array}$ & $\begin{array}{c}35.1 \pm 22.0 \\
(9-73) \\
32.6 \pm 24.2 \\
(12-76) \\
13.5 \pm 12.2 \\
(0-34) \\
11.6 \pm 5.8 \\
(0-22) \\
9.1 \pm 8.1 \\
(1-23) \\
.1 \\
\ldots\end{array}$ & $\begin{array}{c}1561 \pm 725 \\
(439-2773) \\
1344 \pm 1003 \\
(335-2857) \\
362 \pm 324 \\
(0-873) \\
339 \pm 161 \\
(0-609) \\
359 \pm 338 \\
(35-1036) \\
3.8 \\
\ldots\end{array}$ & $\begin{array}{c}5.9 \pm 2.1 \\
(2.0-9.0) \\
5.5 \pm 3.2 \\
(2.5-12) \\
3.6 \pm 3.4 \\
(0-12.3) \\
2.9 \pm 1.5 \\
(0-5.4) \\
2.6 \pm 1.7 \\
(1.2-5.6) \\
\ldots \\
\ldots\end{array}$ & $\begin{array}{c}7.5 \pm 2.9 \\
(2-12) \\
7.9 \pm 2.5 \\
(6-12) \\
4.9 \pm 2.5 \\
(3-10) \\
5.5 \pm 2.0 \\
(3-10) \\
5.4 \pm 1.4 \\
(3-8) \\
4.5 \pm 1.8 \\
(1-6)\end{array}$ \\
\hline
\end{tabular}

Note.-First line each group contains mean and SD; numbers in parentheses are ranges of measured values.

$(P<.05$ or $P<.1)$ with planting density and light intensity (Oohata and ShIDEI 1971). Claims by Oohata and Shidei (1971) and Whitney (1976) concerning the constancy of branching ratios were based on analyses of variance which test the importance of environmental differences relative to the variation within environments. Thus, they might have failed to find significant differences when real differences existed. The probability of type-II error is high when the probability of type-I error is low (SokAL and RoHLF 1969), especially with small samples or when variability of $R b$ was high between trees in the same environment, e.g., cottonwood (table 1), apple and birch (BARKER et al. 1973), and sugar maple (STEINGRAEBER et al. 1979).

High correlations between vigor and the number of lateral shoots formed on a terminal branch (which determines its $R b_{S_{\mathrm{I}} / S_{\text {II }}}$ ) have been well documented by Champagnat (1965). Vigor of shoot growth declines with increasing age and size of trees (BORCHERT 1976), and an age-related decline in the $R b_{\mathrm{S}_{\mathrm{I}} / \mathrm{S}_{\mathrm{II}}}$ is thus to be expected in older trees (compare discussion of Tabebuia below). In the divaricate shrub Aristotelia fruticosa, TomuInson (1978) showed how a decline in bifurcation ratio may occur during the transition from the juvenile to the adult phase because of a transition from a monopodial to a sympodial growth pattern and an increase in branching symmetry.

In summary, bifurcation ratios appear to be neither constant for all trees of a given species nor within crowns of individual trees at any given time or throughout the life of a tree.

The symmetrical branch system of Tabebuia rosea

While branch systems of most trees are markedly asymmetric, as reflected in relatively high Strahler bifurcation ratios (TOMIINSON 1978), branching in saplings of Tabebuia rosea DC. (Bignoniaceae), a tropical tree common throughout Central America, is symmetrical. Tabebuia saplings form initially a single, unbranched stem; then growth of the terminal bud is arrested, and shoot growth continues during the same flush by the symmetrical outgrowth of two opposite, lateral buds, giving rise to a dichasial ramification (sympodial branching; figs. 1, 3). The plane of subsequent bifurcations is perpendicular to that of the previous one.

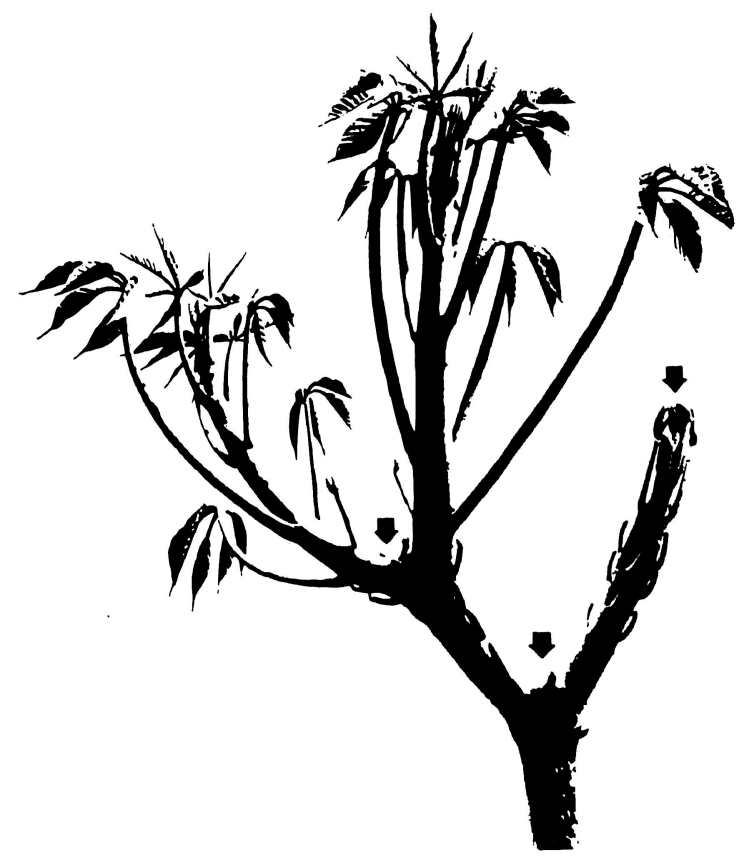

FIG. 3.-Dichasial, sympodial branching in Tabebuia rosea. Arrows indicate arrested terminal buds. Lateral branches continue growth on left, but not right, branch. 
In terms of the botanical ordering system, the number of segments per order will be

$$
S_{n}=2^{n} \text {, where } n=\text { order number, }
$$

and the bifurcation ratio

$$
R b_{S n / S n-1}=2^{n} / 2^{n-1}=2 .
$$

In this special case the order number is identical with the number of branch generations and, for temperate trees forming only one shoot generation per year (e.g., Rhus typhina), equal to the tree's age in years (fig. 4). The semilogarithmic plot of segment number against order is a straight line (fig. 2), which not only provides the descriptive information above but also permits the prediction that, with increasing size (order number), the number of terminal segments-and, hence, leaf area-increases exponentially. The plot thus constitutes a mathematical model describing the branch system and permitting predictions as to the dynamics of its future development.

If the branching system of Tabebuia is ordered by the Strahler system, the semilogarithmic plot for any tree will yield a straight line representing a state description of the tree (fig. 2). If consecutive stages in the branching system of the same tree or trees of different size, i.e., of different maximum order number, are to be graphed, a series of lines with slope $R b$ will result (fig. 2). In contrast to a dynamic model, such a series of state models is not well suited for predictions concerning the future development of a branch system. In terms of practical usage, description of tree development by the Strahler system would require renumbering all branches after each growing season.

The predictions of the mathematical description of branching in Tabebuia (fig. 4) can be tested by comparing them with the state description of $R$. typhina, a temperate tree with dichasial, sympodial branching (fig. 4; data of J. WHITE from HaLLÉ et al. [1978]). These data show that the exponential increase in segment number begins to decline at a rather low order number and then levels off. In other words, "growth" of the number of segments in this branching system, like that of all other biological growth processes, is actually described by a sigmoid, not an exponential, curve, and branching ratios change (decline in the botanical, increase in the Strahler system) as trees increase in size and decline in growth vigor. Symmetrical branch systems thus become asymmetrical during tree development. In Tabebuia this asymmetry is also reflected in increasing differences in the diameters of segments of equal botanical order (fig. 1).

The linear relationship between the logarithm of Strahler segment number and order (fig. 2) is, in essence, an inverse representation of the exponential increase in terminal segments characteristic of trees as of any other geometric series (fig. 4). Because of its lack of isomorphy with botanical trees, this plot obscures the fact that the exponential increase of terminal branches in trees is limited, and order numbers higher than 7 are rarely observed (WILson 1966). Furthermore, while an increase in terminal branch number by $50 \%$ or $100 \%$ will profoundly

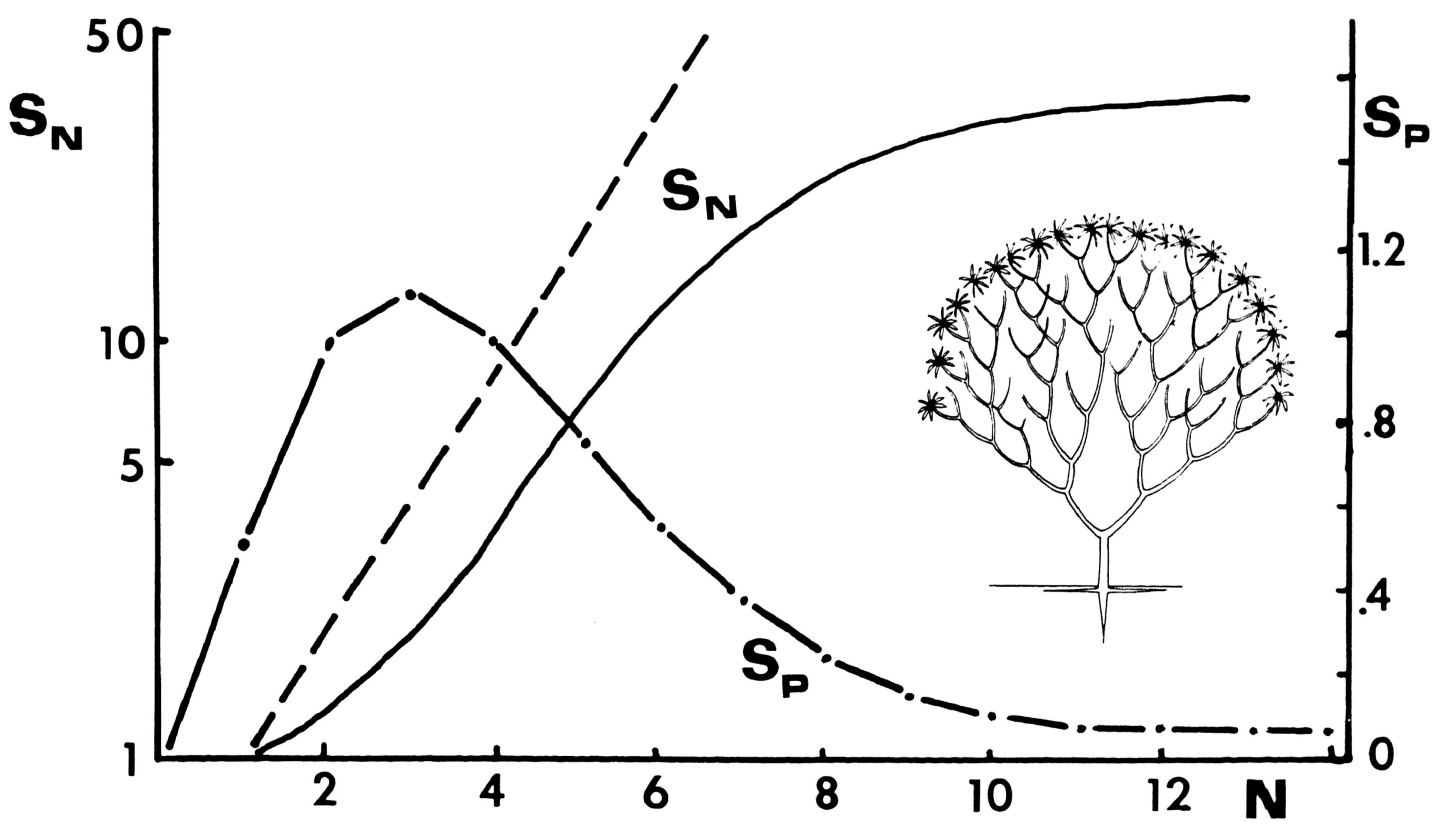

FIG. 4.- Theoretical (- - ) and observed (- $\longrightarrow$ increase in branches per order $\left(S_{N}\right)$ in the sympodial branching system of Rhus typhina (data of J. WHITE from Hallé et al. [1978]) and relative surface area per terminal branch $\left(s_{p}\right)$ as a function of increasing botanical order number $(N)$ on the surface of a symmetrical, sympodial branch system $(-\cdots-)$. Insert: Section through a sympodial branch system (Messersmidea argentia from HaLLÉ et al. [1978]) showing increase in number of unbranched terminal shoots of higher order and hemispherical monolayer of terminal leaf rosettes. 
affect the functional capacities of a tree and change the $R b_{S_{I} / S_{I I}}$ significantly, because of the enormous reduction in scale of low Strahler order segment numbers, such a variation will barely be noticeable in a semilogarithmic plot and will affect the mean $R b$ only slightly. Finally, the number of low-order Strahler segments increases continuously during growth of a tree, but the highest order number will be augmented only rarely. Every time this occurs, the plot will "wag its tail"; i.e., the curve will jump in the part representing higher order numbers as a consequence of the renumbering of these segments (McMaHon and KronaUER 1976).

The sigmoid curve (fig. 4) describes the increase in segment number as a function of order number or time, but it cannot explain the increasing asymmetry of the branch system or the timing of the end of the exponential growth phase. The following considerations will show that such a reduction in terminal branch number must occur sooner or later in tree development, if the branching system is to be optimized for the effective display of photosynthetic surfaces to incoming light. Let us assume that, in a tree such as Tabebuia, all segments are of the same length $l$, and all terminal branches-as well as the leaf rosettes they bear-are arranged in an (isochronic) hemisphere forming a monolayer of leaves (fig. 4). The radius of this hemisphere will be

$$
r_{H}=l \cdot n \text { (where } n=\text { botanical order number) (6) }
$$

and its surface

$$
\begin{gathered}
s_{H}=0.5 \cdot 4 \cdot r_{H}^{2} \cdot \pi, \\
s_{H}=2 \cdot \pi \cdot(l \cdot n)^{2}, \\
s_{H}=k \cdot n^{2} .
\end{gathered}
$$

The partial surface area $s_{p}$ on this hemisphere available for the display of leaves on each terminal branch (fig. 4) will then be ([4] and [8]):

$$
s_{p}=s_{H} / S_{n}=k \cdot n^{2} / 2^{n} .
$$

The curve representing this function (fig. 4) reaches its maximum at order 3 , declines to less than onefifth of the maximum by order 8 , and then approaches zero. As leaf-supporting branches constitute a nonproductive investment of energy, the optimum branch system would be one in which the fewest branches would support a leaf surface covering the entire hemisphere. Consequently, the larger the leaves borne by the terminal branches, the earlier should the exponential increase in segment number end. In $R$. typhina the decline in exponential increase of terminal branches takes place around order 4 or 5 (fig. 4), and in other temperate trees botanical order numbers greater than 7 occur rarely, if at all.

Modeling of branch systems by the method of
HoNDA (1971) has empirically led to similar results. To avoid unnaturally dense branching and excessive overlap of leaves in their model of Terminalia branching, FisHER and HONDA (1977) had to introduce special rules limiting bifurcation of higher order branches. In a theoretical study of branch interaction within simulated branch systems of several tree species, HoNDA, Tomirnson, and Fisher (1981) found that bifurcation ratios changed with increasing order number of simulated trees and were not suited for comparing botanical trees. Likewise, a set of branching rules creating a rather realistic branching pattern of Tabebuia at lower order numbers produces unnaturally dense branching at higher orders $(>8)$, unless restrictions to branching are introduced (fig. 5) (HONDA and BORCHERT, unpublished). The causes for this and other age-related changes in the pattern of tree development have been sought in the correlative changes within trees associated with age-dependent increase in size and complexity (BORCHERT 1976, 1978), but we know nothing about the specific mechanisms which determine cessation of branching in some and its continuation in other terminal branches (fig. 3).

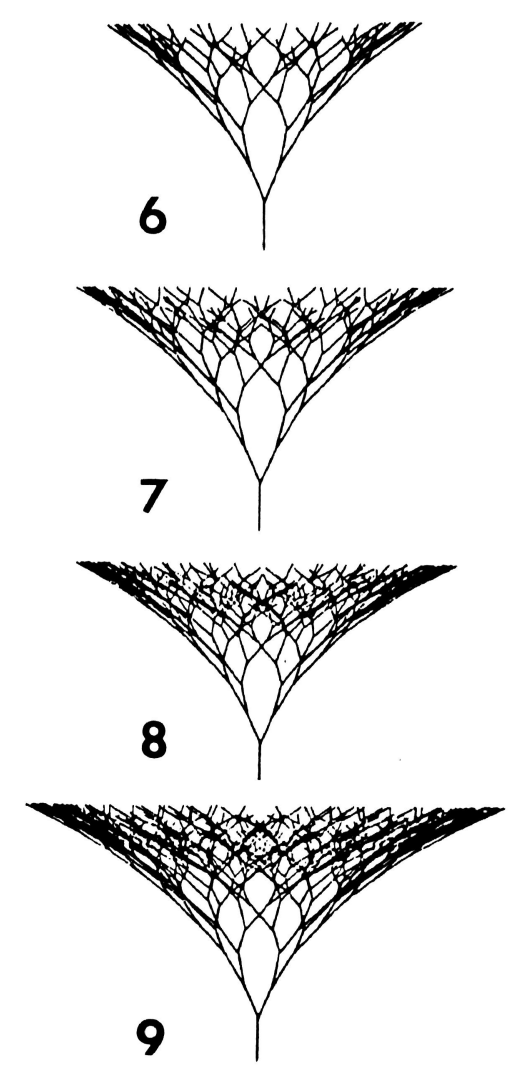

Fig. 5.-Simulation of the branch system of Tabebuia rosea by the method of Honda (1971) from order $N=6$ to $N=9$. Branching angles: $25^{\circ},-25^{\circ}$. Ratio of branch length between consecutive orders: 0.8 . 


\section{Conclusions and prospectus}

The accumulated evidence sheds serious doubt on the postulated usefulness of branching ratios as a tool for quantification of tree branching. However, such ratios are more basically problematic because they represent just one basic property of branching systems, whether these are rivers, or lungs, or botanical trees. They completely ignore the information which confers specificity to tree structure, i.e., the information determining the architectural model of a tree. Differences between ortho- and plagiotropic branches, or differentiation of branches into long or short shoots, are not accounted for in branch ordering or branching ratios; yet these structural features strongly determine and characterize the functional and developmental potentials of a branch, as well as overall tree architecture (HALLÉ et al. 1978). If different architectural models represent different adaptive strategies, then any morphometric method ignoring the specificity of tree form is, a priori, incapable of providing an understanding of the specific structure-function relationship characteristic of a certain architectural model. For similar reasons, McMahon and KronaUer (1976) concluded that any ordering system which does not keep track of the actual physical dimensions of a tree cannot be used to investigate the principle of its mechanical design.

To increase the amount of morphometric information, measurements of branching ratios have been supplemented by data on mean segment length per order, diameter per segment, frequency distributions of first order branches, and other parameters (BARKER et al. 1973; McMaHon and KronaUer 1976; StEINGRAEBer et al. 1979); but a basic study as to what morphometric information is really necessary and sufficient to characterize the adaptive geometry of a tree has not been undertaken. Most of the structural criteria used by HALLÉ et al. (1978) in the taxonomy of architectural models might be required. In a deterministic computer model, HoNDA (1971) generated small trees of rather natural appearance by using variation in segment length as a function of increasing (natural) order in conjunction with the branching angle, which provides information concerning the spatial arrangement of branch segments. He simulated the architecture of two trees, Terminalia catappa and Tabebuia rosea (FISHER and HoNDA 1977) (fig. 5). When the simulated branch system of Terminalia was optimized for maximum effective leaf area, the model predicted branching angles and ratios of branch lengths similar to those observed in nature (HoNdA and Fisher 1978, 1979; Fisher and HoNdA 1979a, 1979b). However, one basic problem associated with the analysis and understanding of large trees persists in this deterministic model: As soon as trees surpass a certain, relatively small size, tree structure ceases to be predictable by deterministic branching rules. Extrapolation from small to large branching systems requires the prediction of sizedependent structural changes by stochastic rules which allow for phenotypic variation, yet conserve the specific architectural model of a tree. The difficulties of establishing such rules are identical to those encountered in all attempts to model or simulate a complex, hierarchical system from the bottom up, i.e., to deduce properties of such a system from information obtained via the reductionist analysis of a small subsystem at a low level of organization.

\section{Acknowledgments}

The experimental part of this study was supported by NSF grant DEB 7724616. This paper is based on a contribution to the symposium, Mathematical Trees in Principle and Practice, held under the auspices of the Maria Moors Cabot Foundation for Botanical Research at the Harvard Forest, Petersham, Massachussets, in May 1979. Discussions at this symposium greatly helped to clarify the ideas presented here. We are much indebted to Dr. P. B. Tomlinson, Harvard University, and Dr. J. B. Fisher, Fairchild Botanical Garden, Miami, for critically reviewing the manuscript and making valuable suggestions for improvement.

\section{LITERATURE CITED}

Barker, S. B., G. Cumming, and K. Horsfield. 1973. Quantitative morphometry of the branching structure of trees. J. Theoret. Biol. 40:33-43.

BORCHERT, R. 1976. The concept of juvenility in woody plants. Acta Hort. 56:21-36.

- 1978. Feedback control and age-related changes of shoot growth in seasonal and nonseasonal climates. Pages 497-515 in P. B. Tominson and M. H. ZrmmermanN, eds. Tropical trees as living systems. Cambridge University Press, Cambridge.

Borchert, R., R. Laushman, and G. E. Glass. 1981. Water stress and growth of cottonwood saplings. Pages 136-148 in H. E. Garketr and G. S. Cox, eds. Proceedings of the Third Central Hardwood Forest Conference. University of Missouri Press, Columbia, Mo.
Champagnat, P. 1965. Physiologie de la croissance et de l'inhibition des bourgeons: dominance apicale et phénomènes analogues. Rameaux courts et rameaux longs. Problèmes physiologiques. Pages 1106-1171 in W. RuHLAND, ed. Encyclopedia of plant physiology. Vol. 15, pt. 1. Springer, Berlin.

Fisher, J. B., and H. Honda. 1977. Computer simulation of branching pattern and geometry in Terminalia (Combretaceae), a tropical tree. Bот. GAz. 138:377-384.

- _ 1979a. Branch geometry and effective leaf area: a study of Terminalia branching pattern. I. Theoretical trees. Amer. J. Bot. 66:633-644.

- $1979 b$. Branch geometry and effective leaf area: a study of Terminalia-branching pattern. II. Survey of real trees. Amer. J. Bot. 66:645-655. 
Halle, F., R. A. A. Oldeman, and P. B. Tomlinson. 1978. Tropical trees and forests. An architectural analysis. Springer, Berlin.

HARRIS, R. J. 1975. A primer of multivariate statistics. Academic Press, New York.

Hollander, M., and D. A. Wolfe. 1973. Nonparametric statistical methods. Wiley, New York.

Honda, H. 1971. Description of the form of trees by the parameters of the tree-like body: effects of the branching angle and the branch length on the shape of the tree-like body. J. Theoret. Biol. 31:331-338.

HondA, H., and J. B. Fisher. 1978. Tree branch angle: maximizing effective leaf area. Science 199:888-890.

- 1979. Ratio of tree branch lengths: the equitable distribution of leaf clusters on branches. Proc. Nat. Acad. Sci. $76: 3875-3879$.

Honda, H., P. B. Tomlinson, and J. B. Fisher, 1981. Computer simulation of branch interaction and regulation by unequal flow ratios in botanical trees. Amer. J. Bot. 68:569585.

Horn, H. 1971. The adaptive geometry of trees. Princeton University Press, Princeton, N. J.

HorTon, R. E. 1945. Erosional development of streams and their drainage basins: hydrophysical approach to quantitative morphology. Bull. Geol. Soc. Amer. 56:275-370.

LEOPOLD, L. B. 1971. Trees and streams: the efficiency of branching patterns. J. Theoret. Biol. 31:339-354.

McMahon, T., and R. E. Kronauer. 1976. Tree structures: deducing the principle of mechanical design. J. Theoret. Biol. 59:443-466.
Miller, J. B. 1978. Living systems. McGraw-Hill, New York. Millington, W. F., and W. R. Chaney. 1973. Shedding of shoots and branches. Pages 149-204 in T. T. KozLowskI, ed. Shedding of plant parts. Academic Press, New York.

Oohata, S., and T. ShIDEI. 1971. Studies on the branching structure of trees: I. Bifurcation ratio of trees in Horton's law. Jap. J. Ecol. $21: 7-14$.

Picketr, S. T. A., and J. S. KempF. 1980. Branching patterns in forest shrubs and understory trees in relation to habitat. New Phytol. 86:219-228.

SokAl, R. R., and F. J. RohLF. 1969. Biometry. W. H. Freeman, San Francisco.

Steingraeber, D. E., L. J. Kascht, and D. H. Franck. 1979. Variation of shoot morphology and bifurcation ratio in sugar maple (Acer saccharum) saplings. Amer. J. Bot. $66: 441-445$.

Strahler, A. N. 1957. Quantitative analysis of watershed geomorphology. Trans. Amer. Geophys. U. 38:913-920.

Tominsson, P. B. 1978. Some qualitative and quantitative aspects of New Zealand divaricating shrubs. New Zealand J. Bot. 16:299-309.

Uylings, H. B. M., G. J. Smith, and W. A. J. Veltman. 1975. Ordering methods in quantitative analysis of branching structures in dendritic trees. Advance. Neurol. 12 :247-254.

WhitNEy, G. G. 1976. The bifurcation ratio as an indicator of adaptive strategy in woody plant species. Bull. Torrey Bot. Club $103: 67-72$.

Wilson, B. F. 1966. Development of the shoot system of Acer rubrum L. Harvard Forest Paper 14. 21 pp.

ZimmermanN, M. H. 1978. Hydraulic architecture of some diffuse-porous trees. Can. J. Bot. 56:2286-2295. 